\title{
Economic Advantages by CAD/CAM Use in Compound with Production Data Organization
}

\author{
Arndt Richter \\ EXAPT Systemtechnik GmbH, Aachen \\ info@exapt.de
}

\begin{abstract}
Current developments concerning the manufacturing industry cause also new and additional requirements for the use of CAD/CAM systems. It is represented that beside the technological further development especially the virtualization of the planning processes has significant influence. Furthermore the local technical consideration of the CAD/CAM process is not sufficient. The general organization in the factory has to be considered. The integrated consideration of the physical specification of the production means with the organization of the production resources ensures a long-term economic success for the $\mathrm{CAD} / \mathrm{CAM}$ use. To realize this target the possibilities and advantages are described in this article.
\end{abstract}

Keywords: CAD/CAM, multi-task machine tool, automatic feature recognition, 3-5-axis-milling, virtualization, simulation, tool management, production data management, blank part actualization, tool sequence optimization, 3D tool models, production resources, communication platform.

\section{Introduction}

In the manufacturing industry a highly interesting upheaval takes place presently. Not only new production means with new technical possibilities, modified production structures with out- or insourcing or whole production transfer to other production areas, but especially also new approaches for the networking of information about the complete production operations is meant. These new approaches, also summarized under the slogan Industry 4.0, will allow among others

- the further growing together or the parallelism of product development and product design processes

- the better linkage of virtual and real world of the production as well as

- the penetration of the communication in all areas of planning, preparation and execution of the production.

Thereby the digitalization of the production sequences turned out as one of the greatest driver of innovation. The constantly improving junction of the virtual and real production will increasingly be important for an efficient production. Therewith also for classical processes of production planning, e.g. CAD/CAM planning or production 
data and tool management new tasks arise, which only lead to a better economic representation in interaction of all components.

CNC-controlled machine tools always require digital data, that means digitized geometry, technology and organizational data as input for the machine tool control. In the course of the development powerful CAD/CAM systems originated from the simple NC programming, which include under the aspect of the overall system machine tool - clamping device - tool - workpiece extensive planning functions. But for the efficient use of these systems more and more digital data of the process control, production means and production resources are required to create safety and reliable results. Systems for the production organization as PLM-, tool management- or MESsystems have been established parallel to the geometric and technical oriented CAD/CAM systems. All systems require similar, partially equal data concerning the content, to generate the demanded planning results. The required data are managed locally and in general not compatible because of different development approaches and development targets. From the point of view of an economic production the great challenge is to obtain the continuity and consolidation of the organizational and technical oriented systems.

\section{Challenges for CAD/CAM Solutions}

The requirements of the manufacturing industry concerning the CAD/CAM systems are very versatile and characterized by different factors.

In the machine tool sector currently dominate especially multi-task machines, which enable a mix of processes in many different ways. Therewith it is possible to machine workpieces completely on one machine. However, this causes a more and more complex kinematics of the machine tool and in the large machine tool construction the increasingly dominating influence of change-over units like angle heads, facing heads etc.

Directly associated with the development of the machine tool sector are the innovations in the field of tool technology. New production engineering and production strategies can be realized with according modern tools. Examples are High Speed Cutting or High Performance Cutting, but also adaptive machining strategies and combined procedures, e. g. of classical cutting methods in combination with laser-use.

On the other hand new production organizational strategies have been established in the production. An unbroken change from the batch production "on stock" to a client-specific production as well regarding the order number as also the constructive specification can be recorded. Therewith associated are always smaller batch sizes or piece number 1 , whereby the efficient single production gets the primacy. Under this aspect preformed workpieces can only be used in a limited way, whereas the machining "from one block", e.g. also the production of prismatic parts from bar material, is becoming increasingly important. 
From the mentioned strategic developments in the production and organization area permanently new changing and more extensive challenges result for the CAD/CAM planning. So e. g. the rate of material removal increases permanently and as a result the expenditure for planning, preparation and program generation for the CNC machine clearly increases. The complexity of programming tasks is also growing. The expenditure per program and the number of necessary $\mathrm{NC}$ programs per time unit continue to rise drastically and therewith also the CAD/CAM planning is asked to gain more efficiency.

In addition to the pure technical program generation also the requirements concerning the reliability and safety of the created results and the documentation of the planning results increase continuously. Only with clearly improved and more efficient planning methods the requirements from complex production systems can be realized with the requested quality.

Because of the reduction of batch sizes the start-up time increases also drastically in relation to the entire production time. It is required that additionally to a fast and flexible program supply for the machines all necessary production-relevant documents and information are provided reliably. Only this way it is possible to reduce the start-up time drastically. The requirement to minimize the start-up time is emphasized because the low batch sizes require an enhanced flexibility in the production. This has also an impact on re-planning in the production engineering which has to be realized CAD/CAM system supported. A corresponding system support enables to realize the requirements regarding a flexible production with low batch sizes.

Current and future production facilities cannot be seen without a look to the overall organizational process in the factory. Essential requirements to CAD/CAM systems arise because of these integrated considerations from the product development to the CNC machine including the data, resource and quality management. The integrated consideration of all areas is decisive for a successful economic production.

\section{Technical Aspects}

The NC preparation has to react fast and flexibly to the often changing requirements from the manufacturing. The results have to be provided with high safety so that the following process steps can be created reliably. For that current CAD/CAM systems offer an lot of possibilities so work fast and efficient. 3D-CAD workpiece models, designed regarding the production technical aspects, are the starting point. This statement is important because for an integrated CAD/CAM planning the responsibility for the measure is based on the CAD model. Therefore pure $3 \mathrm{D}$ visualization models of workpieces are not suitable for production engineering. Using an automatic, in modern CAD/CAM systems integrated, feature recognition the required parameters for the production-oriented processes can be determined quickly. Accordingly adapted systems provide very quick and flexible nearly fully automatic results for an efficient production this way (fig. 1). 


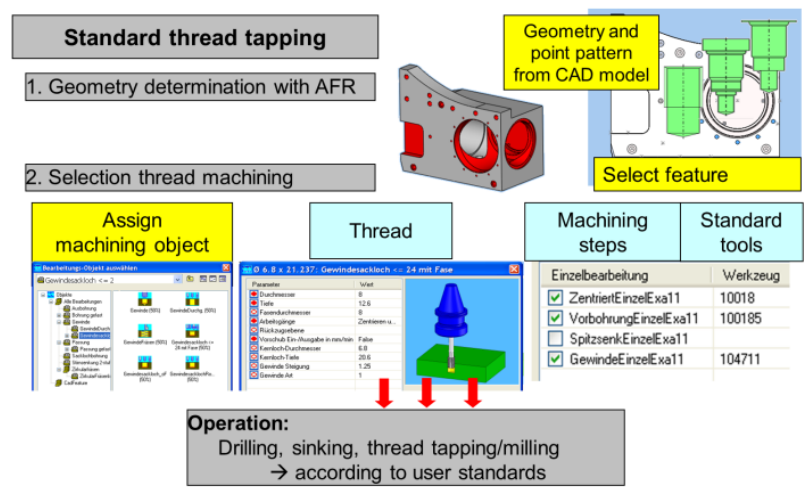

Fig. 1. Automatic feature recognition with automatically generated and client-specific extended machining

Thereby it is important that in addition to standard sequences respective customdesigned machining strategies can be stored to guarantee a know-how protection and also individual company-specific sequences.

For the complete machining of a workpiece with several machining steps in terms of a mix of process with turning, drilling and milling of 2 - 5 axes a dynamic blank part actualization is indispensable. For each machining step the current blank part has to be provided to plan the following machining step with the current geometry data (fig. 2)

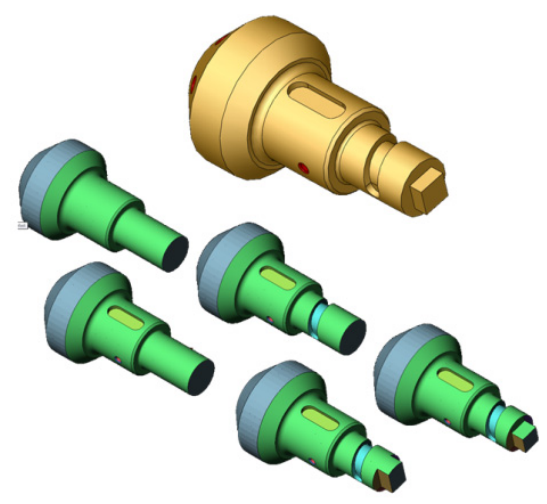

Fig. 2. CAD/CAM planning with blank part actualization in all processes steps

Because of the complexity of the workpiece geometry and the production requirements resulting therefrom it is more and more necessary to use additional sets on machine tools. Only this makes it possible to realize the geometric and qualitative machining requirements. In the large part manufacturing sector such change-over units are absolutely essential, because a relocating of the workpiece is extremely complex, so that the total workpiece has to be machined with one or two settings as 
possible. The use of different angle heads, facing heads etc. is the rule. Large machine tools have additionally different parallel axes so that the tool can approach to the machining point efficiently. For the CAD/CAM planning such machine kinematics are special challenges. Not only the pure machining has to be regarded and the tool paths to be generated but especially the entire question of the use of aggregates have to be considered. This is the consideration of the tool and attachments handling here, especially the head positioning and the head change. A further emphasis is in this context the tool sequence optimization over several machining sides including the use of change-over units.

Beneath the organizational handling of change-over units including the handling of perhaps changing coordinate systems for the several machining sides of different aggregate applications the visualization of the complete process has the highest priority. For the simulation of the machining sequence it is differentiated basically the tool path-related simulation and the machine simulation based on the NC codes. EXAPT has the target to provide a respective detailed simulation already in the planning of the several process steps. This tool path-related simulation including the display of the kinematics of the machine is completed by a removal simulation with integrated collision control, so that the user gets a largely realistic display. The advantage of the immediate visualization of each machining step during the planning stage is that subsequently no corrections have to be made. Basically both simulation strategies complement one another and lead, at correct application, to high efficiency in the production. All aspects of change-over units have to be regarded and visualized in the case of tool path-oriented simulation, naturally. Especially the Placing of the attachments to the workpiece for the processing and the collision check of the attachments with the workpiece, the clamping devices and other machine elements are important (fig. 3).

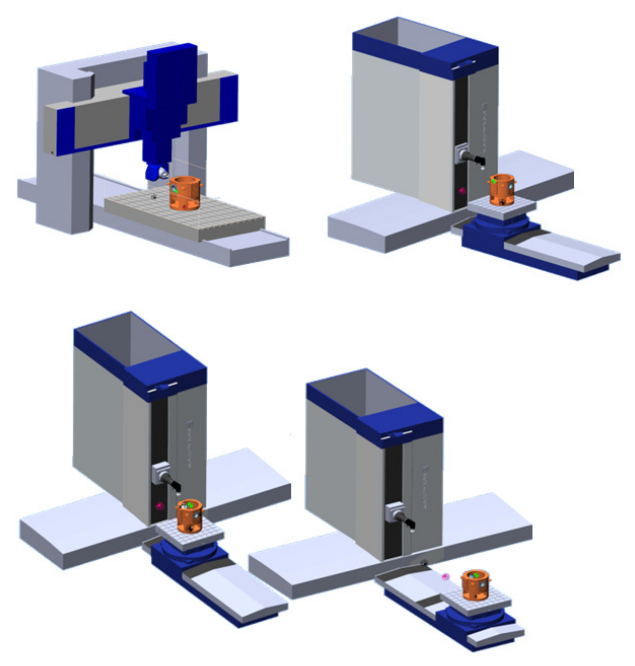

Fig. 3. Simulation of the machine environment based on tool paths 
The specific challenge to CAM systems is for this simulation strategy in the planning phase not only to simulate machine motions but also to display transfer motions of the machine tool e. g. concerning the tool or aggregate change, where the tool is not used. The current EXAPT developments have set broad standards in this area. The entire kinematics of the machine tool can be displayed because of the integration of all movements, also the passive machine movements in the processor, and the range of functions of the postprocessor can be reduced explicitly (fig. 4).

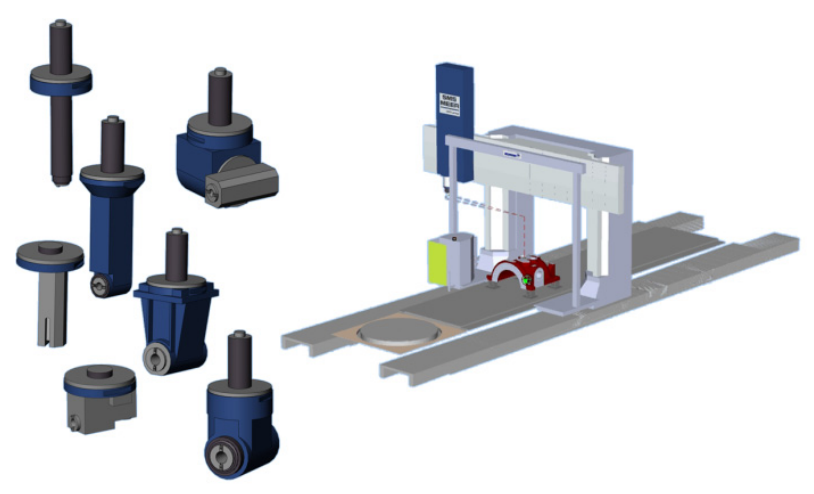

Fig. 4. Large machine tool with tool and attachment change

\section{Organizational Aspects}

Regarding the described complex manufacturing procedures in detail it quickly becomes clear that beside the physical specification of the system machine tool - clamping device - tool - workpiece also the organizational states of the production resources are eminently important. Therewith especially the tools move into the focus.

In addition to the pure technical description of the tool and especially of the cutting edge also the geometric specification of the assembled tool as a 3D model is required for a safe technology-oriented production planning. This is the condition for a safely collision control and an objective visualization of the entire process. There is a need to distinguish in general that concerning the tool geometry has to be differentiated between a physical and a technological tool model. This quickly becomes clear for asymmetric rotatory tools as boring bars (fig. 5).

The technical description of the tool essentially determines the accuracy and therewith the quality of the planning process. Important economic effects are developed additionally by a corresponding organization of the classic tool department. This are not simply questions e. g. of the storage of tool components or the pre-reservation of special tools for the machining but especially the tool logistic and the job oriented scheduling of tool deployment on the shop floor. 

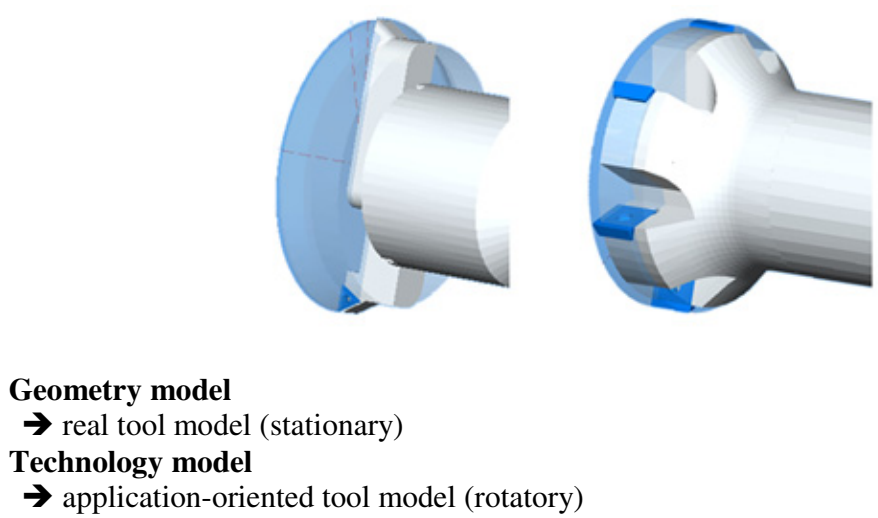

Fig. 5. Appropriate tool data for the NC production

Scheduling of tool deployment thereby comprises the whole process from the tool selection in the CAM process via the generation of orders and the provision of tool data for tool presetting machines up to the transfer of the necessary data to the machine tool. A significant reduction of set-up time can be reached by combining several tool setting orders with an according sequence optimization of the production orders. Thereby the savings are not only in the machine area but also in the tool presetting area, because fewer tools have to be set. Savings up to $30 \%$ of logistic time for tools in relation to normal processes can be verified with this solution. In the comparison between the real set-ups state on the machine with the planned set-up orders are further effects which can be realized with an online communication to the machine tool.

\section{$5 \quad$ Integrated Data Concept between Technique and Organization}

The above-mentioned statements clarify that at every point of the process planning safely and reliable information of the state of the production means and production resources have to be available. Considering only one side of the planning tasks the positive results are ruined by other negative influences. Therefore it is necessary to create a corresponding communication to enable the provision of the relevant data in every process step. This is the only way to ensure that the planning results really correspond to reality. Beside reliability and completeness of the required technical characteristics an according communication platform is extremely important (fig. 6).

This communication platform does not only have the function e. g. to exchange data between a CAD/CAM and a tool management system but has to consider the entire process. 


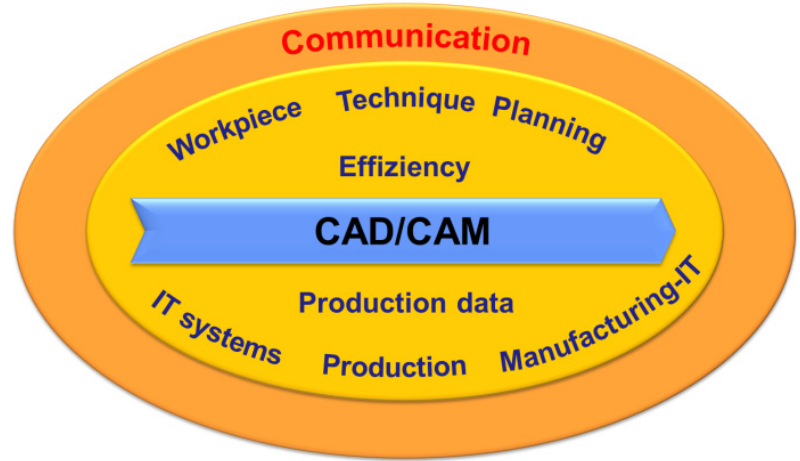

Fig. 6. Technique, organization and communication in the area of the CAD/CAM system

All partners participating in the entire process have to be addressed by suitable communication techniques, e. g. WEB-based solutions, and to be integrated into the general communication. In the area of tool logistic this refers to the questions of communication with different storage systems, the mobile tool identification, the tool presetting or the communication with NC controls. Only this complex logistic consideration makes it possible to obtain the economic effects resulting from this solution. But it is also clear that a corresponding dovetailing of the information is absolutely essential today. With the EXAPT production communication manager EXAPT provides a system which manages a browser-oriented uncomplicated communication with the several stations in the shopfloor. Additionally it is possible provide all production relevant information via respective techniques at every place. Therewith according process sequences can be created more transparent, information can be exchanged immediately and bottlenecks can be displayed transparently more quickly, if necessary.

\section{Conclusions}

It can be seen that an economic CAD/CAM-use requires a high technological performance of the system combined with according simulation techniques for the visualization and control of the results, but additionally the compound to the organization, especially to the tool management, are indispensable. Due to the close connection of $\mathrm{CAD} / \mathrm{CAM}$ and organization the economic success can be realized, especially in terms of small run and client-oriented production. Thereby it is not a matter of point to point connections but by building a communication platform the matter is a strategic orientation of the whole communication technique inside a CAD/CAM compound. 